$\mathrm{ECHO}$.

\title{
Adhesion factors and endothelial growth factor vary with RA type
}

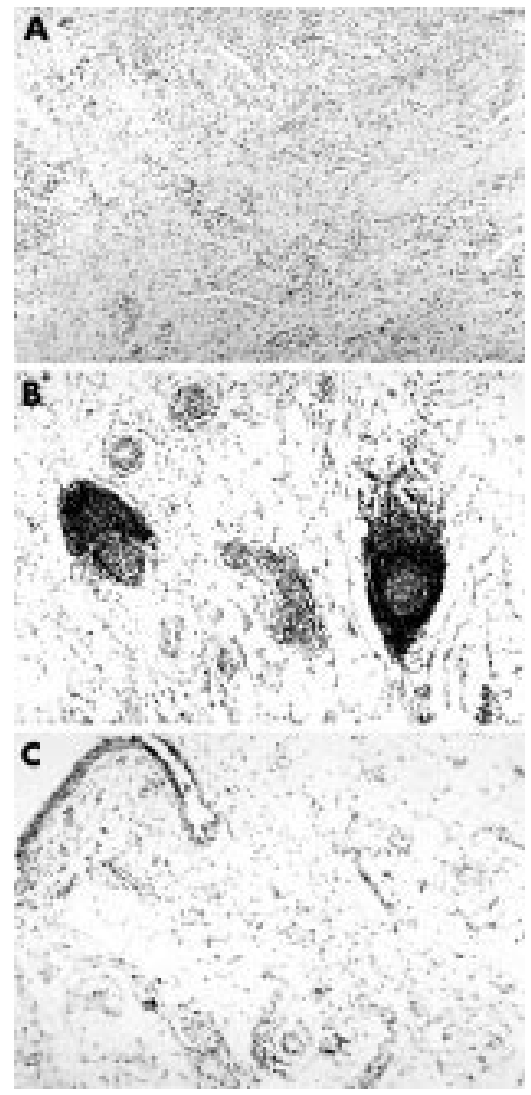

istinct histological forms of rheumatoid arthritis (RA) may respond differently to
treatment as they have different patterns of soluble adhesion factors and vascular endothelial growth factor (VEGF), Polish researchers claim.

Their investigations centred on synovial and serum samples from 42 patients with RA and 32 controls with osteoarthritis (OA). Serum concentrations of soluble intercellular adhesion molecule 1 (sICAM-1), soluble vascular adhesion molecule 1 (sVCAM-1), soluble E-selectin (sE-selectin), and VEGF were significantly higher in patients with RA than OA. All except sE-selectin were much higher for patients with RA showing follicular synovitis (16) compared with patients showing diffuse synovitis (24) and patients with OA. They were also linked with clinical markers for RA, including erythrocyte sedimentation rate, $\mathrm{C}$ reactive protein, number of swollen joints, and $x$ ray evidence indicating more severe disease.

In follicular RA the synovium contained circular aggregates of lymphocytes around a central core and associated with new capillaries. In diffuse synovitis and OA it showed a more uniform spread of mononuclear cells throughout the tissue.

Synovial tissue was taken from patients during hip or knee operations. Serum concentrations of adhesion factors and VEGF were measured by ELISA.

Distinct types of synovitis seem to be found with different patterns of cytokines in the synovium and cytokines and metalloproteinases in serum. Macrophages and fibroblasts migrate to the synovium under the influence of adhesion molecules and increased vascularisation promoted by VEGF, sVCAM, and sE-selectin. Once there their cytokines and metalloproteinases mediate tissue destruction, maybe controlled by lymphocytes.

A Annals of the Rheumatic Diseases 2002;61:804-809.

Typical histopathological appearance of synovial tissue. $(A, C)$ uniform spread of mononuclear cells in RA or OA, respectively; (B) follicular arrays in RA. Original magnification $\times 100$.

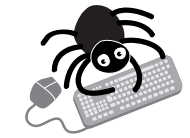

Please visit the Molecular Pathology website [www.molpath.com] for link to this full article.

\section{Fetal DNA may spark Sjögren's syndrome}

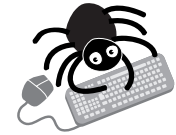

Please visit the Molecular Pathology website [www. molpath.com] for link to this full article.
- jögren's syndrome (SS) may have its origins in fetal DNA from an earlier pregnancy. A molecular study has shown for the first time male fetal DNA in the inflammatory lesions. Male specific DNA occurred in biopsy specimens from affected salivary glands in a significant proportion of mothers with SS who had one or more sons. It also showed up in cells from lung aspirates from a significant proportion of those mothers with interstitial lung lesions. The sequence was absent from mononuclear cells from the blood and all samples from the controls. Women with SS and no sons did not have the sequence, apart from two-explained by a previous abortion and a blood transfusion. All three biopsy specimens tested by fluorescence in situ hybridisation (FISH) were positive.

Fifty six women with SS were tested. Blood was obtained from 27, biopsy specimens from 42 , and cells from bronchiolar lavage fluid from nine. The controls were eight healthy women volunteers with no previous pregnancy, 10 women with mucocoele but no SS, and 15 women with other lung diseases, respectively. Male specific DNA was detected by nested PCR and FISH.

Male DNA, probably from fetal cells in the mother's circulation, occurs in skin lesions due to other autoimmune diseases, though a universal link is disputed. Lesions of SS and graft versus host disease are similar, suggesting a similar disease mechanism.

Larger scale testing is needed; also microchimerism as described here, but with female fetal cells, might feature in pathogenesis.

A Annals of the Rheumatic Diseases 2002;61:1041-1046 